\title{
Searching for high-redshift centimeter-wave continuum, line and maser emission using the Square Kilometer Array
}

\author{
A. W. Blain ${ }^{\mathrm{a}}$, C. Carilli ${ }^{\mathrm{b}}$, J. Darling ${ }^{\mathrm{c}}$

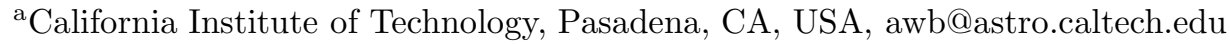 \\ bNRA, Socorro, NM, USA, ccarilli@nrao.edu \\ ${ }^{c}$ OCIW, Santa Barbara Street, Pasadena, CA, USA, darling@ociw.edu
}

We discuss the detection of redshifted line and continuum emission at radio wavelengths using a Square Kilometer Array (SKA), specifically from low-excitation rotational molecular line transitions of CO and HCN (molecular lines), the recombination radiation from atomic transitions in almost-ionized hydrogen (radio recombination lines; RRLs), $\mathrm{OH}$ and $\mathrm{H}_{2} \mathrm{O}$ maser lines, as well as from synchrotron and free-free continuum radiation and $\mathrm{HI}$ 21-cm line radiation. The detection of radio lines with the SKA offers the prospect to determine the redshifts and thus exact luminosities for some of the most distant and optically faint star-forming galaxies and active galactic nuclei (AGN), even those galaxies that are either deeply enshrouded in interstellar dust or shining prior to the end of reionization. Moreover, it provides an opportunity to study the astrophysical conditions and resolved morphologies of the most active regions in galaxies during the most active phase of star formation at redshift $z \sim 2$. A sufficiently powerful and adaptable SKA correlator will enable wide-field three-dimensional redshift surveys at chosen specific high redshifts, and will allow new probes of the evolution of large-scale structure (LSS) in the distribution of galaxies. The detection of molecular line radiation favours pushing the operating frequencies of SKA up to at least $26 \mathrm{GHz}$, and ideally to $40 \mathrm{GHz}$, while very high redshift maser emissions requires access to about $100 \mathrm{MHz}$. To search for LSS the widest possible instantaneous field of view would be advantageous.

\section{Introduction}

The processes that established the properties of the populations of galaxies we see in the Universe today have begun to be probed in detail only during the last decade (see summaries at long and short wavelengths by Smail et al. 2002 and Steidel et al. 2003 respectively). Information about the evolution of galaxies out to redshifts $z \sim 5$ and beyond is now being gathered as an ever increasing rate. Multiwavelength studies have been confirmed as vital to investigate the nature of the processes at work in forming galaxies (e.g. Alexander et al. 2003), and radio observations have played a major part in these developments (e.g. Chapman et al. 2003b). However, existing radio telescopes are only sensitive enough to detect the most luminous galaxies (brighter than at least a few $10^{12} \mathrm{~L}_{\odot}$ ) at redshifts greater than two. A much more sensitive radio instrument would provide a better match to the capabilities of the
James Webb Space Telescope $(J W S T)^{1}$, the Atacama Large Millimetre Array (ALMA) ${ }^{2}$ and future 30-m-class ground-based telescopes such as the TMT under study. ${ }^{3}$

Key questions that remain in galaxy formation include the evolution of the luminosity function of galaxies prior to the apparent peak of starformation activity at $z \simeq 2-3$ (Madau et al. 1996; Blain et al. 1998; Chapman et al. 2003a; Giavalisco et al. 2004; Steidel et al. 2004). The maximum luminosity of galaxies at such early epochs is still little known, although very luminous examples have been found (Becker et al. 2001; Fan et al. 2004). The evolution of the space densities and luminosities of galaxies extending through the newly established epoch from the end of reionization at $z \simeq 6.5$, to its beginning at $z \simeq 20$, as highlighted by the recently

\footnotetext{
$\overline{{ }^{1} \text { http://ngst.gsfc.nasa.gov/ }}$

${ }^{2}$ http://www.alma.nrao.edu/

${ }^{3}$ http://www.astro.caltech.edu/observatories/tmt/
} 
detected Gunn-Peterson effect and cosmic microwave background (CMB) polarization observations respectively (Spergel et al. 2003) is entirely unresolved by existing observations.

Because reionization has almost no effect on the opacity of the Universe to radio waves, a very sensitive radio telescope could be tremendously useful for finding and revealing the processes at work in the galaxies that reionized the intergalactic medium (IGM) at $z>6$. The role of feedback from star formation and AGN activity in modifying the stellar formation rate, populations and AGN accretion is also little known, but must be sensitive to the masses and densities of galaxies, that can be probed using radio line observations deep into dust obscured regions. Radio continuum emission can provide insight into the power of supernovae and the density of the interstellar medium (ISM) in these early objects. The low opacities of both the IGM and ISM to radio and mm-wave line emission ensures that this radiation could provide information on the masses and excitation conditions right into the cores of the very earliest galaxies that formed.

Alongside studies of the properties of individual forming galaxies, the richness of large-scale structure (LSS) in the three-dimensional distribution of galaxies has been resolved in unprecedented detail, both at moderate redshifts (Blanton et al. 2003), and out to greater distances (Venemans et al. 2002; Adelberger et al. 2003; Blain et al. 2004; Coil et al. 2004). Overdensities in the distribution of galaxies found near known extremely luminous objects have also been resolved (Brand et al. 2003; Stevens et al. 2004). In the future, radio spectroscopy could have a key role to play in resolving the three-dimensional structures traced by very large numbers of normal galaxies over wide fields at high redshifts, building on redshift determinations now possible using the Westerbork WSRT ${ }^{4}$ and the Greenbank Telescope (GBT). ${ }^{5}$ Radio spectroscopy has the advantage of tracing the bulk of the ISM in galaxies, and so accurate relative velocities between nearby galaxies can be obtained, without concern for the

${ }^{4}$ http://www.astron.nl/p/observing.htm

${ }^{5}$ http://www.gb.nrao.edu/GBT/GBT.shtml effects of winds and outflows to confuse relative velocities (Shapley et al. 2003), and blur out velocity space distortions that reveal the relaxation of clusters of galaxies.

\subsection{Existing radio observations}

The faintest galaxies that can be detected detected in the radio continuum using the NRAO VLA have been studied for almost 20 years (Donnelly, Partridge \& Windhorst 1987), and the $\mu \mathrm{Jy}$ population continues to promote important insight into the galaxy formation process to the present day, providing an extinction independent, high-resolution view of the evolution of galaxies (Haarsma et al. 2000; Chapman et al. 2003b; Cowie et al. 2004). However, spectroscopy at cm wavelengths using existing instruments remains difficult, owing to very restrictive correlator bandwidths, and only single very luminous objects can be targeted for radio spectroscopy at the VLA (e.g. Walter et al. 2003).

The availability of radio observations has proved to be very valuable in the interpretation of the nature of new populations of dust-enshrouded galaxies that are relatively difficult to detect in all but the deepest images at optical and wavelengths (Smail et al. 1997, 2002; Frayer et al. 2004), but nevertheless have luminous dust continuum emission at mid- and far-infrared(IR) and submillimeter (submm) wavelengths (Altieri et al. 1999; Smail, Ivison \& Blain 1997; Smail et al. 2002; Bertoldi et al. 2002, 2004; Greve et al. 2004). These galaxies that contribute a significant fraction of the luminosity of all galaxies at high redshifts (Blain et al. 1999). Radio observations have been crucial in pinpointing the positions of these galaxies with sufficient accuracy to enable optical spectroscopy, changing them from an interesting but anecdotal population of objects with uncertain properties into a well studied sample of galaxies (Chapman et al. 2002, 2003a, 2004; Smail et al. 2003; Blain et al. 2004).

All observations of distant galaxies in the radio waveband benefit from negligible extinction, and well-designed interferometers offer both naturally fine angular resolution and wide instantaneous fields of view. With sufficient collecting area the instrumental sensitivities are excellent (at the $\mu \mathrm{Jy}$ 
level), and the key requirement for spectroscopy is a sufficiently powerful correlator to provide the bandwidth to conduct redshift surveys across the whole degree-scale instantaneous field of view. ${ }^{6}$ This class of performance would be tremendously powerful for probing high-redshift LSS, as the largest scales of order $100 \mathrm{Mpc}$ manifested in the galaxy distribution correspond to about 3-4 deg on the sky at high redshifts, while individual distant galaxies subtend angles of order 0.5 arcsec. Resolved images and three-dimensional spectra of all the members of a forming high-redshift cluster of galaxies could thus be obtained in only a few pointings of SKA.

The flexibility and power of a correlator capable of wide-field spectroscopy could also be very adaptable to multi-beam-forming observations of widely-separated known targets or for continuum surveys over several fields of view.

The prospects for detecting the radio synchrotron and free-free continuum emission from distant, low-luminosity galaxies are excellent while existing radio surveys reach down to of order $10 \mu \mathrm{Jy}$ at $1.4-\mathrm{GHz}$, the proposed sensitivity of the SKA corresponds to a depth of $80 \mathrm{nJy}$ RMS in $24 \mathrm{hr}$ over a field that could be as wide as $1.2 \mathrm{deg}^{2}$. Arcsec-scale resolution will be essential to avoid confusion at the faintest levels. Note that the detection rate of galaxies at redshifts greater than unity of about $10^{4} \mathrm{hr}^{-1}$ is unprecedented in astronomy.

A huge task remains to find redshifts for these faint radio galaxies, many of which, and perhaps some of the most interesting examples, may not be easily accessible to optical or near-IR spectroscopy, either because of a lack of emission lines, or to extreme distance and faintness. Fine angular resolution ensures that the problem is not as severe for SKA-detected galaxies as for those selected at far-IR or submm wavelengths. The discovery images at shorter wavelengths have relatively poor angular resolution, including the forthcoming avalanche of many millions of new galaxies expected to be discovered

\footnotetext{
$\overline{{ }^{6} \mathrm{~A} 12 \text {-m scale element }}$ at $1 \mathrm{GHz}$ provides a 1.5 -deg-wide field of view, while a $100-\mathrm{km}$ baseline corresponds to a resolution of 0.06 arcsec.
}

by Spitzer Space Telescope (Spitzer) $)^{7}$ following its August 2003 launch (Werner, Gallagher \& Irace, 2004), and the fainter galaxies to be detected using the future 3.5-m Herschel Space Observatory after $2008^{8}$. In part, ground-based integral field unit spectrographs can locate galaxies when positions are known less accurately than a few arcsec, but to compile large samples and substantially complete redshift catalogs, techniques that rely on neither accurate positions nor optical telescopes will probably be required. Several types of diagnostic lines are available to be probed at radio wavelengths, both the centimeter and millimeter bands, and these could offer a breakthrough with future radio telescopes. The SKA could be the workhorse instrument to unlock the multiwavelength Universe, much as the VLA has been in the last decade for the existing small samples of the most luminous high-redshift galaxies (Smail et al. 2002; Chapman et al. 2003a,b, 2004).

The advantage that a radio interferometer, with appropriately small individual elements has over the ALMA mm/submm-wave interferometer is a potentially much greater instantaneous field of view, and a greater chance of serendipitous detection of line emission at very high redshifts (Carilli \& Blain 2002). ALMA can carry out the necessary observations in molecular lines (Blain et al. 2000), but only for a relatively tiny instantaneous field of view. Operating at much longer wavelengths, a radio array can cover a much wider field, while using longer baselines to maintain angular resolution, detecting molecular transitions, recombination and maser lines in relatively low excitation gas.

\section{Line emission mechanisms}

\subsection{Low-excitation molecular lines}

The fuel for star formation in both the local and distant Universe is dense molecular gas, cooled by optically thin emission from molecular and metal fine-structure lines, mechanisms which require prior enrichment of the ISM in the galaxies. In the earliest objects molecular hydrogen would be the cooling mechanism (Rees \& Ostriker 1977;

\footnotetext{
${ }^{7}$ http://www.spitzer.caltech.edu/
}

${ }^{8}$ http://herschel.jpl.nasa.gov/ 
Sokasian et al. 2004). Hence, the second generation of star formation, potentially deep in the epoch of re-ionization at $z \simeq 6-15$ should generate powerful $115-\mathrm{GHz} \mathrm{CO}(1-0)$ line emission, while the densest regions could excite and emit 88.6-GHz $\mathrm{HCN}(1-0)$ radiation.

Working in the radio waveband, rotation transitions between only the lower- $J$ states of polar molecules are available for observations. The most promising molecules are $\mathrm{CO}$ and $\mathrm{HCN}$ (from which emission is likely to be an order of magnitude fainter than $\mathrm{CO}$ ). Other molecules like $\mathrm{HCO}^{+}$might be as promising as $\mathrm{HCN}$ for highredshift studies. Depending on the maximum frequency of the SKA bands, $\mathrm{CO}$ and $\mathrm{HCN}$ could be observed for all redshifts greater than about 1.6 and 0.95 respectively (for an upper frequency limit of $44 \mathrm{GHz}$ matched to the limit of atmospheric transparency). For very high-redshifts $(z>9.5)$, should metal-enriched galaxies exist there, higher-excitation lines up to $\mathrm{CO}(4-3)$ might also be detectable, redshifted into this potential operating range of the SKA extending to about $40 \mathrm{GHz}$. At such very high redshifts, the increased CMB temperature $(\simeq 30 \mathrm{~K})$ would allow higher- $J$ level transitions to occur, as the relevant rotational levels of $\mathrm{CO}$ and $\mathrm{HCN}$ will be excited. Note, however, that the lines will not be made more luminous by scattering off higherenergy CMB photons.

Molecular line emission from the $115-\mathrm{GHz}$ $\mathrm{CO}(1-0)$ transition has been detected from one of the most distant QSOs currently known (Walter et al. 2003), and from other very luminous, high-redshift galaxies (Carilli et al. 2002, 2004; Lewis et al. 2002; Greve et al. 2003; Solomon et al. 2003). The 10 to few- $100 \mathrm{~km} \mathrm{~s}^{-1}$ width of detected lines is likely to provide a good estimate of the velocity dispersion of the gas in the system in which it is detected (Sheth et al. 2004; Tacconi et al. 2005), and if sub-arcsec resolution is available, then an accurate mass can be derived for organized systems.

Out to $z \simeq 20$ at the likely start of reionization (Spergel et al. 2003), CO(1-0) emission could be detected at a frequency of $5-8 \mathrm{GHz}$, if sufficiently luminous galaxies were present. This is a speculative, but not impossible goal for a future gener- ation of very sensitive radio telescopes.

Fig. 2 presents the number of $\mathrm{CO}$, finestructure and radio recombination lines expected at 4 chosen example $4-\mathrm{GHz}$ wide SKA observing bands. The calculations follow those outlined in Carilli \& Blain (2002) and Blain et al. (2000). The results have been updated to include relatively small shifts in the fitting parameters required by the new redshift distribution for submm/radio-selected galaxies determined by Chapman et al. (2003a; 2004), and to take account of the post-WMAP cosmological parameters $\left(\Omega_{\mathrm{m}}=0.27, \Omega_{\Lambda}=0.73\right.$ and $\left.H_{0}=71 \mathrm{~km} \mathrm{~s}^{-1}\right)$ : see Knudsen et al. (2005).

\section{Galaxies in absorption: $\mathrm{CO}$ and $\mathrm{HI}$}

With excellent resolution in both the spectral and spatial domains, SKA absorption line spectroscopy should be possible, both against highredshift radio-loud AGN (e.g Wiklind \& Combes 1998; Murphy, Curran \& Webb 2003), or distant transient GRB sources (see Piro et al. 2002). While there is hope that bright radio galaxies can be found at $z>5$ (Jarvis et al. 2001; Furlanetto $\&$ Loeb 2002), transients would have the advantage of an effectively unlimited redshift, and thus the presence of $\mathrm{CO}$ absorption at rest $115-\mathrm{GHz}$ along a large number of independent lines of sight to $z \simeq 20$ could provide direct measurements of the earliest epoch at which etals became present in the ISM of galaxies. In principle, sufficiently bright illuminating sources could be used to detect absorption due to a wide variety of more complex molecules. The redshift of the first molecules is likely to be the same as the epoch of the first illuminating beacons, and so it presents the opportunity to make a consistency check of the first star-formation activity.

Transient radio pulses should last for hours to days in their restframes, and then be time dilated to arrive as signals with durations of days to months, and so the SKA could in principle detect absorbers without requiring very rapid response (Furlanetto \& Loeb 2002). At $100 \mathrm{MHz}$, the instantaneous field of view is large, and so in a deep image the possibility of monitoring a constantly varying set of faint transients can be forseen. The imaging quality from long baselines 
to avoid source confusion is an important issue. Arcsec resolution, and $1000 \mathrm{~km}$ baselines would be required.

\subsection{Radio recombination lines (RRLs)}

RRLs have been sought observationally for 30 years, but have only rarely been useful for revealing practical extragalactic astrophysics (see Mohan, Anantharamaiah, \& Goss 2002), owing to their low intrinsic luminosities (see Oh \& Mack 2003). The sensitivity of the SKA offers to change this situation dramatically. Maser modification of the line intensities, which can only increase their detectability, is likely for transitions at lower frequencies than the $\mathrm{H} 50 \alpha$ line at $51.1 \mathrm{GHz}$. Note that at higher frequencies, the lines $\mathrm{H} n \alpha,{ }^{9}$ and $\mathrm{H}(n+1) \alpha$ are separared by a difference in frequency that can be calculated using the formula in the footnote, and is of order $3 \mathrm{GHz}$ at $50 \mathrm{GHz}$. In the calculations shown in Fig. 2 the $\mathrm{H} 50 \alpha$ line is assumed to include $1.9 \times 10^{-9}$ of the total power emitted by the galaxy (Seaquist, Kerton \& Bell 1994), and other lines are assumed to be populated thermally, with luminosities proportional to $\nu^{2}$. The frequency separation of adjacent RRLs is of order $6 \%$ of the line frequency, and so at least 2 and usually 3 lines should be present in any $4-\mathrm{GHz}$ window from high redshifts, even out to $40 \mathrm{GHz}$. Hence, although faint, RRLs could provide a direct, single-shot redshift indicator for any star-forming galaxies detected with enough power to have a signal-to-noise ratio greater than about 100 in the radio continuum over the typical $100 \mathrm{~km} \mathrm{~s}^{-1}$ line width of the galaxy. This corresponds to a continuum flux density of about $0.4 \mathrm{mJy}$ in a 24-hour integration at a frequency of $1.4-\mathrm{GHz}$, or to an ultraluminous galaxy at $z \simeq 2$. By detecting multiple lines, the exact set of transitions $(n)$ being observed can be determined without any ambiguity, leading to a very accurate redshift for the source, with a centroid of $\pm 30 \mathrm{~km} \mathrm{~s}^{-1}$, indicating a redshift accuracy of only $\Delta z \simeq 0.0003$ at $z \simeq 2$. The relative intensities of the lines can then be used to check on possible maser activity and the physical condi-

\footnotetext{
${ }^{9}$ This transition is between the $n+1$ and $n$ quantum states, at a frequency close to $3.289 \times 10^{15}\left[n^{-2}-(1+n)^{-2} \mathrm{~Hz}\right.$ (Lyman- $\alpha$ is $\mathrm{H} 1 \alpha$ in the same terminology
}

tions within the warm almost-ionized, recombining gas in the source. The optically thin emission from RRLs should be related accurately to the ionization rate in the ISM and/or intergalactic gas around the galaxy, while the associated neutral atomic and molecular gas could be probed by $\mathrm{HI}$ and $\mathrm{CO}(1-0)$ emission in sufficiently deep observations using ALMA or SKA once the redshift was known. Taken together, over fields that are many tens of arcmin in extent, the SKA should be able to trace the history of processing and enrichment of gas in resolved detail in RRLs over the most active epochs of galaxy formation at $z \simeq 2$ 3 .

\subsection{HI emission}

The emission of 21-cm $\mathrm{H}$ hyperfine structure radiation is discussed more extensively elsewhere (van der Hulst et al. 2004; this volume), but it will provide a useful way to probe the mass of neutral gas in distant galaxies. With a sufficiently long baseline and integration time, it may be possible to resolve the internal spatial structure of galaxies, allowing accurate modeling of the mass distribution within the inner baryonic part of both primordial gas clouds and metal-rich galaxies at high redshifts. Existing facilities can detect the most massive objects in Hi emission to only moderate redshifts (e.g. Zwaan et al. 2001), and in absorption in more luminous distant example (De Breuck et al. 2003). A far more sensitive telescope, with a wide spectral band could use redshifted Hi to trace reionization (Furlanetto \& Loeb 2002), and image with sufficient spatial resolution to probe galaxies' LSS at $z \simeq 3$ over a representative volume, targeting a 5-deg-wide swath of sky simultaneously with a $10-\mathrm{m}$ antenna size.

\subsection{Masers}

Rich series of transitions in $\mathrm{OH}$ and $\mathrm{H}_{2} \mathrm{O}$ molecules can be driven into population inversions to generate powerful maser emission (Darling, Goldsmith \& Giovanelli 2002; Darling \& Giovanelli 2003). Especially important for observations out to high redshifts are likely to be the $1665 / 1667-\mathrm{MHz}$ OH maser lines and 1612/1720$\mathrm{MHz}$ conjugate lines (Darling 2004). Any tele- 
scope that is efficient at detecting Hi emission from low redshifts, can also detect these lines, and a very sensitive interferometer like SKA should be able to detect them out to high redshift (Darling et al. 2004). The intense illumination required to drive the gas into a population inversion could be readily produced by dense knots of star formation activity in even the most distant systems, and so the $0.1-1 \mathrm{GHz}$ band could be very promising for selecting and measuring the redshifts of distant galaxies. This is a regime where the lower frequency coverage of the SKA can make significant contributions, with a modest requirement on correlator bandwidth as compared with the demands of a CO-based system (Townsend et al. 2001). The expectation that a 2 -line maser spectrum would be seen prevents confusion with intervening lower-redshift Hi emission, and provides direct confirmation of the redshift identification at a single frequency (Briggs 2000).

An added, speculative use of the physically simple, small regions where maser emission arises is that the relative frequencies of different maser transitions can be measured very accurately, and are sensitive to any changes in the value of the fine structure constant $\alpha$ out to moderate and high redshifts (Darling 2003). Highly controversial remains possible evolution of the fine-structure constant has been suggested based on optical observations of QSO absorption lines (e.g. Murphy, Webb \& Flambaum 2003; Chand et al. 2004). $\mathrm{OH}$ absorption measures and weak maser lines offer to probe this evolution very accurately. The conjugate maser strength is $1 \%$ of the continuum, and can be detected out to the maximum redshift of bright background galaxies (Darling 2004).

Maser lines provide alternatives to another radio method using 1.4-GHz Hi radiation (Carilli et al. 2000; Carilli \& Taylor 2000), and one which may be less prone to systematic effects (see Curran et al. 2004; this volume).

The relative spatial positions of any $\mathrm{OH}$ maser emission within a $200 /(1+z) \mathrm{MHz}$ bandwidth of the SKA when combined with the frequency of the maser line could provide a very valuable tracer of the kinematics of the maser emission spots with a galaxy, and could possibly be used to trace accelerations in multi-epoch observations.

\section{Continuum emission}

At present the VLA is able to detect the synchrotron continuum emission from the combined supernovae remnants in galaxies out to $z \simeq 3$, but only from those galaxies with star-formation rates in excess of several $100 \mathrm{M}_{\odot} \mathrm{yr}^{-1}$ (Chapman et al. 2002). The necessary surveys reach $1-\sigma$ sensitivities of several $\mu \mathrm{Jy}$. Most have been conducted at $1.4 \mathrm{GHz}$, although deep 5- and 8-GHz observations have been made; multiband radio data is valuable, as a flatter or steeper spectral index than $f_{\nu} \propto \nu^{\alpha}$ with $\alpha \simeq-0.6$ could be used to spot faint AGN amongst the sample of high-redshift star-forming galaxies. Measurement of the continuum slope could also provide additional astrophysical information (van der Hulst et al. 2004; this volume).

The emission from synchrotron sources is expected to be reduced at higher redshifts, as the intensity of the CMB radiation increases as $(1+z)^{4}$, and provides a loss mechanism by scattering the relativistic electrons responsible for powering synchrotron emission, and effect that becomes important when the energy density in the CMB $\left(U_{\mathrm{CMB}}\right)$ matches that in the local ISM magnetic field $U_{\mathrm{B}}$. This is likely to occur for quiescent spiral galaxies until $z \simeq 1$; however, at higher redshifts inverse Compton cooling will dominate synchrotron radiation as a mechanism to remove energy from relativistic electrons, leading to shorter lifetimes, and a departure to the radio-quiet side of the tight radio-far-IR correlation (Condon et al. 1992)

This is not true however for the most luminous compact nuclear starburst galaxies with star formation rates $>100 \mathrm{M}_{\odot} \mathrm{yr}^{-1}$ in regions only several $100 \mathrm{pc}$ in extent (Downes \& Solomon 1998). In these systems the energy density in the magnetic field is almost three orders of magnitude larger than that in the Milky Way disk (Carilli \& Taylor 2000), in which case $U_{\mathrm{CMB}}$ only becomes relevant at $z>7$. However, compact nuclear starbursts raise a different, related problem: the energy density in the IR radiation field $\left(\mathrm{U}_{\gamma}\right)$ from the starburst itself is larger than that in the magnetic field. This means that inverse Compton cooling should remove the synchrotron emitting electrons on fairly short timescales $(\leq$ 
$10^{5} \mathrm{yr}$ ), and accentuates the question: why do low-redshift nuclear starbursts follow the radiofar-IR correlation? There is a large body of literature on this issue, but as yet no resolution. Hence, we trade one problem - inverse Compton losses off the microwave background at high redshift - for a second - inverse Compton losses off the starburst infrared radiation field at all redshifts.

The densest, most energetic regions of star formation may be able to sustain synchrotron emission to higher redshifts, but during the extremely distant epoch of reionization at $z \simeq 6$-20, only short-lived GRB and AGN jets will be detectable in synchrotron emission.

Free-free continuum emission will always remain detectable, however, whenever high-mass star formation is taking place. While the intensity of nebular free-free emission is less than that of synchrotron emission in nearby galaxies (Condon 1992), a sufficiently sensitive telescope could detect the associated continuum sources out to $z \simeq 20$. Excellent angular resolution would be required to identify individual sources.

Fig. 1 shows the expected count of very faint star-forming galaxies and radio-quiet AGN expected at a variety of frequencies from 0.5 to $8 \mathrm{GHz}$, including the expected effect of $\mathrm{CMB}$ emission suppression, with and without the contribution of free-free emission, based on models described in more detail by Blain et al. (2002) and Carilli \& Blain (2002). The effect of free-free emission at boosting the number of faint, highredshift galaxies is clear, especially when observing at the higher SKA frequencies.

Note that it will never be possible to detect thermal dust emission from very high redshift galaxies using cm-wave telescopes, as the dust temperature cannot fall below that of the CMB. At the highest redshifts $(z>10)$, the Kcorrection that is so beneficial for detecting distant galaxies at submm wavelengths (Blain \& Longair 1993; Blain et al. 2002) is prevented from operating further, and free-free emission is expected to be the most powerful source of radiation at all restframe frequencies of order 100$300 \mathrm{GHz}$ that will be probed by SKA at the highest redshifts (Blain et al. 2002).

\section{Gravitational lensing}

With the potential to resolve the shapes of high-redshift galaxies, and to determine their redshifts using RRLs over a large field in a single very deep exposure, it could be possible to use SKA in deep integrations to generate a cosmic shear map that highlights the mass in foreground structures. The 0.1 -arcsec spatial resolution is necessary to resolve high-redshift galaxies, and is thus essential for this type of study. Without knowing the nature of the morphology and internal spatial distribution for the emission from numerous distant radio sources in detail it is difficult to assess the true capabilities (Schneider et al. 2000; Blake et al. 2004, this volume). However, the chances of success are very high, as SKA imaging quality and depth available should be sufficient. Both subarcsec resolution and $\mu \mathrm{Jy}$ sensitivities are absolute requirements for this goal, while a wide instantaneous field of view would be very useful to generate a sufficiently large number $\left(\simeq 10^{5}\right)$ of detected galaxies with accurate shapes (Fig. 1).

The most massive foreground galaxies, ellipticals out to $z \simeq 1$, will be abundant in every SKA pointing. In their vicinity will be the faint radio images of background galaxies distorted and magnified by the ellipticals. These images will appear in all SKA pointings that are sufficiently deep ( $\simeq 10$ nJy RMS) to have a source density of order $10^{6} \mathrm{deg}^{-2}$ No dedicated observing time will be necessary for these observations. These massive, non-star-forming foreground galaxies are not expected to be strong radio sources, with the possible exception of point sources in their nuclei. and so the contrast between lens and image should be very high, in contrast to optical studies of galaxy-galaxy lensing for which the emission from the foreground lens dominates. The images whose morphologies indicate the strongest distortion and thus the highest magnification would be excellent candidates for resolved studies one by one using ALMA.

\section{Conclusions}

Wide-field wideband spectra at radio wavelengths offer several unique opportunities for 
studying the evolution of both galaxies themselves and their large-scale structure. Radio recombination lines at low redshifts, highly redshifted molecular lines from close to the end of recombination, and absorption lines against sources prior to the end of recombination are all previously barely explored phenomena that will be relatively easy to investigate using an SKA. The possibilities for its exploitation of these effects would advocate the emphasis of the following instrument design features:

1. For all surveys of high-redshift galaxies, resolutions of 1 arcsec are essential to avoid confusion, and ideally resolutions better than 0.1 arcsec would allow substructure to be probed within the distant objects, imposing a minimum baseline requirement of $150 \mathrm{~km}$ for frequencies as high as $40 \mathrm{GHz}$, and an Earth-sized baseline at the lowest frequencies.

2. To detect low-excitation $\mathrm{CO}$ and $\mathrm{HCN}$ emission from cold molecular gas in distant galaxies it is necessary to push the frequency range of the SKA up towards the $40 \mathrm{GHz}$ atmospheric cutoff, to ensure that the radiation from the most active phase of galaxy evolution at $z \simeq 2-3$ can be probed.

3. For LSS surveys, the most powerful, most easily reconfigurable wide-band correlator is required. The ability to simultaneously correlate a spectrum over a large fraction of the receiver bandwidth would be a very useful goal, providing the capability for line surveys with great depth in redshift.

4. For all deep surveys it is more desirable to have a larger number of smaller antennae for the same total collecting area in order to maximize the instantaneous field of view. This would maximize the efficiency of the telescope, and highlight some of the faintest yet most interesting galaxies for study using ALMA, JWST and future 30-m optical/near-IR ground-based telescopes.

To summarize, an instrument with $10^{4} 10-\mathrm{m}$ apertures, a maximum baseline of $1000 \mathrm{~km}$, and a very powerful correlator would be extremely valuable for studying galaxy evolution at radio wavelengths. 


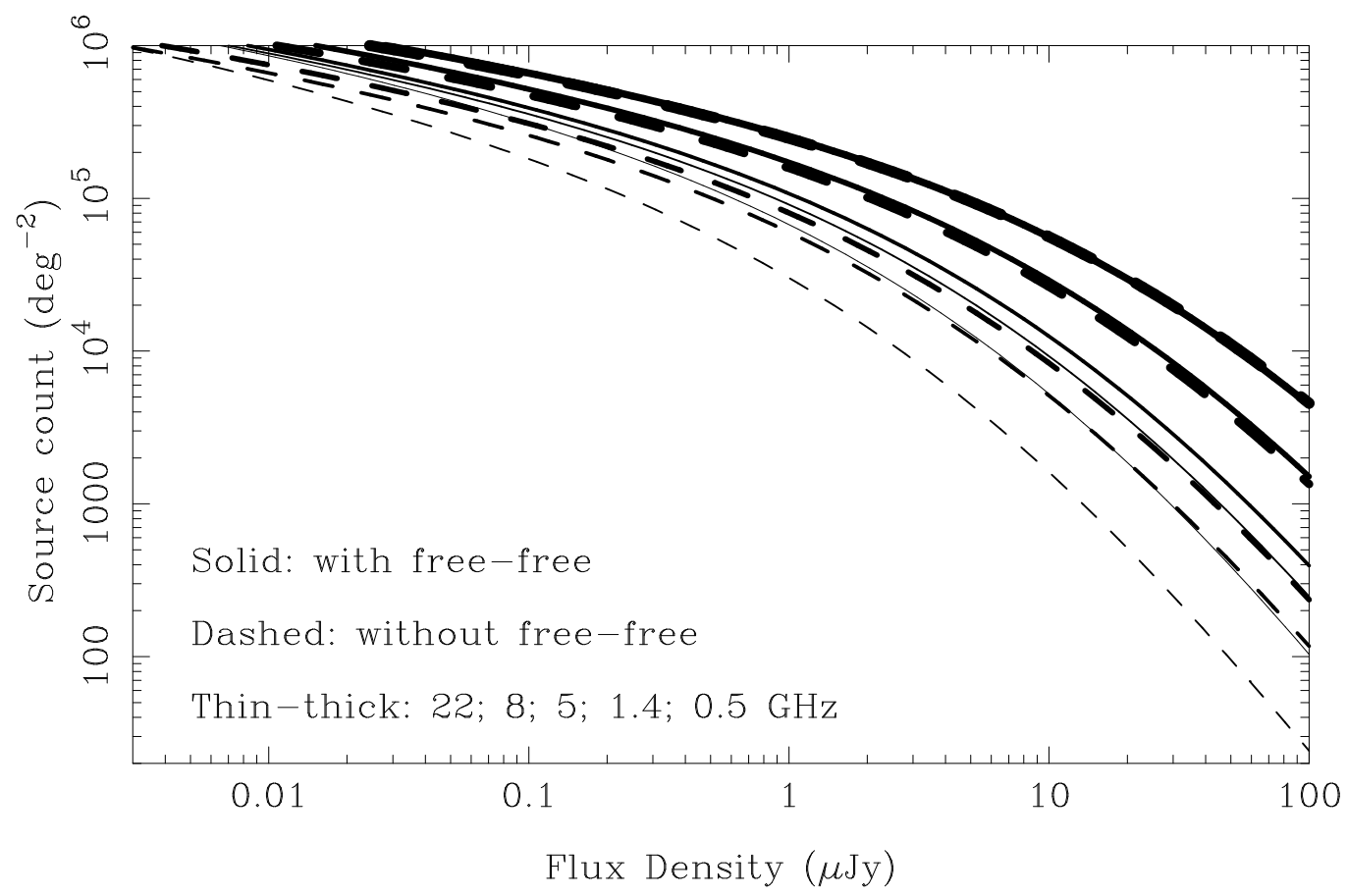

Figure 1. The integral count $N(>S)$ of continuum galaxies expected at extremely faint radio flux density levels $S$, for several different SKA wavelengths, based on a model in which the radio population is extrapolated from dusty star-forming galaxies (see Blain et al. 2002). Existing surveys reach detection limits no fainter than $10 \mu \mathrm{Jy}$ at $1.4 \mathrm{GHz}$. The maximum flux is below the cutoff at which the count of radio-loud AGN falls beneath that of star-forming galaxies in existing 1.4-GHz surveys. There should be a very large sample of continuum-emitting galaxies for the SKA to detect. 

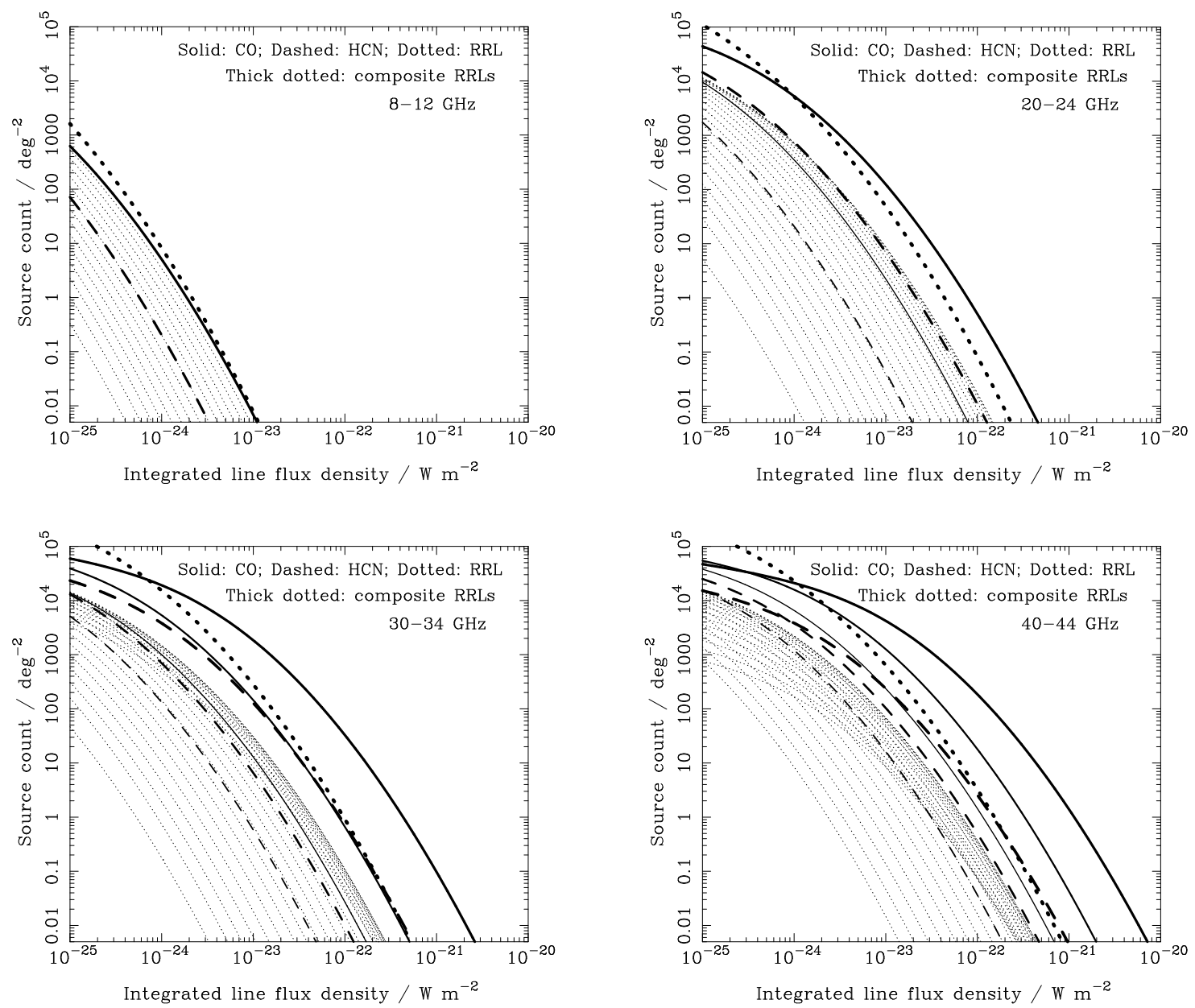

Figure 2. The count of line-emitting galaxies expected out to redshift $z=10$ in four higher-frequency potential SKA bands, each $4 \mathrm{GHz}$ wide. The thickest solid and dashed lines represent the (1-0) transitions of $\mathrm{CO}$ and $\mathrm{HCN}$ respectively; progressively thinner lines of the same style represent higher $J$ transitions $(J+1 \rightarrow J)$. In the lower frequency bands the (2-1) and (3-2) transitions are not redshifted far enough to be detectable. The higher-excitation RRLs at higher frequencies than $n=50(51.1 \mathrm{GHz})$ are shown (dotted lines), In each band, the dotted lines represent lines in the RRL ladder with lower $n$ values, at the lower left side, starting with $n=19$ : dotted lines with progressively greater values of $n$ appear at the upper right, until the count is maximized for a certain value of $n$, and the count then declines (as shown by the flat low-flux counts for $40-44 \mathrm{GHz})$. At lower excitations $(n>50)$, the effect of masing could boost the RRL line strength, and so the contribution of RRLs could be greater than these results suggest. Note that in the combined total count of galaxies, RRLs (at lower redshifts than the CO and HCN sources) could contribute a comparable number of detections as the molecular lines, and so could provide a very valuable redshift survey at moderate redshifts, especially $z \simeq 1.5$ for the $20-24 \mathrm{GHz}$ band. In a 1-hr integration at $42 \mathrm{GHz}$, a $5-\sigma$ sensitivity of about $1.2 \times 10^{-24} \mathrm{~W} \mathrm{~m}^{-2}$ is expected for a $300-\mathrm{km}_{-} \mathrm{s}^{-1}$ wide line, while at $22 \mathrm{GHz}$ the corresponding value is $2.8 \times 10^{-24} \mathrm{~W} \mathrm{~m}^{-2}$. This corresponds to of order 50 line detections within the 0.03-deg-wide primary beam of a 12-m element SKA in a 1-hr integration, and thus to a viable redshift survey. 
AWB acknowledges support from NSF grant AST-0205937, from the Research Corporation and from the Alfred P. Sloan Foundation. We thank Joy Chavez for compiling data on RRLs during a summer internship at Caltech in 2003, supported by the Caltech Minorities Undergraduate Research Fellowship program (MURF).

\section{REFERENCES}

1. Adelberger, K. L., Steidel, C. C., Shapley, A. E., \& Pettini, M. 2003, ApJ, 584, 45

2. Alexander, D. M., et al. 2003, AJ, 126, 539

3. Altieri, B., et al. 1999, A\&A, 343, L65

4. Becker, R. H., et al. 2001, AJ, 122, 2850

5. Bertoldi, F., et al. 2004, in prep.

6. Bertoldi, F., Menten, K. M., Kreysa, E., Carilli, C. L., \& Owen, F. 2002, Highlights in Astronomy, 12, 473

7. Blain, A. W., Chapman, S. C., Smail, I., \& Ivison, R. J., 2004, ApJ, 611, 725

8. Blain, A. W., Smail, I., Ivison, R. J., Kneib, J.-P., \& Frayer, D. T. 2002, Phys Rep, 369, 111 (astro-ph/0202228)

9. Blain, A. W., Frayer, D. T., Bock, J. J., \& Scoville, N. Z. 2000, MNRAS, 313, 559

10. Blain, A. W., Smail, I., Ivison, R. J., \& Kneib, J.-P. 1999, MNRAS, 302, 632

11. Blain, A. W. \& Longair, M. S. 1993, MNRAS, 264, 509

12. Blake, C., et al. 2004, this volume

13. Blanton, M. R., et al. 2003, ApJ, 592, 819

14. Brand, K., Rawlings, S., Hill, G. J., Lacy, M., Mitchell, E., \& Tufts, J. 2003, MNRAS, 344, 283

15. Briggs, F. H. 2000, Perspectives on Radio Astronomy: Science with Large Antenna Arrays, Proceedings of the Conference held at the Royal Netherlands Academy of Arts and Sciences in Amsterdam on 7-9 April 1999. Edited by M. P. van Haarlem. Published by ASTRON. ISBN: 90-805434-1-1, 340 pages, 2000, p.75 (astro-ph/9910415)

16. Carilli, C. L., Bertoldi, F., Walter, F., Menten, K. M., Beelen, A., Cox, P., \& Omont, A. 2004, in Multiwavelength AGN Surveys. Edited by R. Maiolino and R. Mujica. Published by World Scientific, 8 pages astro-ph/0402573)

17. Carilli, C. L., \& Blain, A. W. 2002, ApJ, 569, 605

18. Carilli, C. L., et al. 2002, AJ, 123, 1838

19. Carilli, C. L., et al. 2001, Physical Review Letters, 85, 5511

20. Carilli, C. L., \& Taylor, G. B. 2000, ApJ, 532, L95

21. Chand, H., Petitjean, P., Srianand, R., \& Aracil, B., 2004, A\&A, submitted (astro-ph/0408200)

22. Chapman, S. C., Blain, A. W., Smail, I. R., \& Ivison, R. J., 2004, ApJ, submitted

23. Chapman, S. C., Blain, A. W., Ivison, R. J., \& Smail, I. R. 2003a, Nat, 422, 695

24. Chapman, S. C., et al. 2003b, ApJ, 585, 57

25. Chapman, S. C., Lewis, G. F., Scott, D., Borys, C., \& Richards, E. 2002, ApJ, 570, 557

26. Coil, A. L., et al. 2004, ApJ, 609, 525

27. Condon, J. J. 1992, ARA\&A, 30, 575

28. Cowie, L. L., Barger, A. J., Fomalont, E. B., \& Capak, P. 2004, ApJ, 603, L69

29. Curran, S. J., et al. 2004, this volume

30. Darling, J., Giovanelli, R., Haynes, M. P., Bolatto, A. D., \& Bower, G. C. ApJL, in press (astro-ph/0408531)

31. Darling, J. 2004, ApJ, in press (astro-ph/0405240)

32. Darling, J. 2003, Physical Review Letters, 91, 011301

33. Darling, J. 2004, ApJ, in press (astro-ph/0405240)

34. Darling, J., Goldsmith, P., Li, D., \& Giovanelli, R. 2003, AJ, 125, 1177

35. Darling, J., \& Giovanelli, R. 2002, ApJ, 572, 810

36. De Breuck, C., et al. 2003, A\&A, 401, 911

37. Donnelly, R. H., Partridge, R. B., \& Windhorst, R. A. 1987, ApJ, 321, 94

38. Downes, D. \& Solomon, P. M. 1998, ApJ, 507, 615

39. Fan, X. et al., 2004, AJ, 128, 515

40. Frayer, D. T., Reddy, N. A., Armus, L., Blain, A. W., Scoville, N. Z., \& Smail, I. 2004, AJ, 127, 728

41. Furlanetto, S. R., \& Loeb, A. 2002, ApJ, 579, 1

42. Giavalisco, M., et al. 2004, ApJ, 600, L103 
43. Greve, T. R., Ivison, R. J., \& Papadopoulos, P. P. 2003, ApJ, 599, 839

44. Greve, T. R., Ivison, R. J., Bertoldi, F., Stevens, J. A., Dunlop, J. S., Lutz, D., \& Carilli, C. L., 2004, MNRAS, submitted (astro-ph/0405361)

45. Haarsma, D. B., Partridge, R. B., Windhorst, R. A., \& Richards, E. A. 2000, ApJ, 544,641

46. Jarvis, M.J., Rawlings, S., Willott, C.J. et al. 2001, MNRAS, 327, 907

47. Knudsen, K. K., et al., 2005, A\&A, submitted

48. Lewis, G. F., Carilli, C., Papadopoulos, P., \& Ivison, R. J. 2002, MNRAS, 330, L15

49. Loeb, A., \& Barkana, R. 2001, ARA\&A, 39, 19

50. Madau, P., Ferguson, H. C., Dickinson, M. E., Giavalisco, M., Steidel, C. C., \& Fruchter, A. 1996, MNRAS, 283, 1388

51. Mohan, N. R., Anantharamaiah, K. R., \& Goss, W. M. 2002, ApJ, 574, 701

52. Murphy, M. T., Curran, S. J., \& Webb, J. K. 2003, MNRAS, 342, 830

53. Murphy, M. T., Webb, J. K., \& Flambaum, V. V. 2003, MNRAS, 345, 609

54. Oh, S. P., \& Mack, K. J. 2003, MNRAS, 346, 871

55. Piro, L., et al. 2002, ApJ, 577, 680

56. Rees, M. J. \& Ostriker, J. P. 1977, MNRAS, 179,541

57. Schneider, P. 2000, Perspectives on Radio Astronomy: Science with Large Antenna Arrays, Proceedings of the Conference held at the Royal Netherlands Academy of Arts and Sciences in Amsterdam on 7-9 April 1999. Edited by M. P. van Haarlem. Published by ASTRON. ISBN: 90-805434-1-1, 340 pages, 2000, p.203

58. Seaquist, E. R., Kerton, C. R., \& Bell, M. B. 1994, ApJ, 429, 612

59. Shapley, A. E., Steidel, C. C., Pettini, M., \& Adelberger, K. L. 2003, ApJ, 588, 65

60. Sheth, K., Blain, A. W., Kneib, J.-P., Frayer, D. T., van der Werf, P. P., \& Knudsen, K. K., 2004, ApJL, in press

61. Sokasian, A., Yoshida, N., Abel, T., Hernquist, L., \& Springel, V. 2004, MNRAS, 350, 47
62. Smail, I., Chapman, S. C., Ivison, R. J., Blain, A. W., Takata, T., Heckman, T. M., Dunlop, J. S., \& Sekiguchi, K. 2003, MNRAS, 342, 1185

63. Smail, I., Ivison, R. J., Blain, A. W., \& Kneib, J.-P. 2002, MNRAS, 331, 495

64. Smail, I., Ivison, R. J., \& Blain, A. W. 1997, ApJ, 490, L5

65. Solomon, P., Vanden Bout, P., Carilli, C., \& Guelin, M. 2003, Nat, 426, 636

66. Spergel, D. N., et al. 2003, ApJS, 148, 175

67. Steidel, C. C., Adelberger, K. L., Shapley, A. E., Pettini, M., Dickinson, M., \& Giavalisco, M. 2003, ApJ, 592, 728

68. Steidel, C. C., Shapley, A. E., Pettini, M., Adelberger, K. L., Erb, D. K., Reddy, N. A., \& Hunt, M. P. 2004, ApJ, 604, 534

69. Stevens, J. A., Page, M. J., Ivison, R. J., Smail, I., \& Carrera, F. J. 2004, ApJ, 604, L17

70. Tacconi, L., Lutz, D., et al. 2005, ApJ, submitted

71. Townsend, R. H. D., Ivison, R. J., Smail, I., Blain, A. W., \& Frayer, D. T. 2001, MNRAS, 328, L17

72. van der Hulst, J. M., et al. 2004, this volume 73. Venemans, B. P., et al. 2002, ApJ, 569, L11

74. Walter, F., et al. 2003, Nat, 424, 406

75. Werner, M. W., Gallagher, D. B., \& Irace, W. R. 2004, Advances in Space Research, 34, 600

76. Wiklind, T., \& Combes, F. 1998, ApJ, 500, 129

77. Zwaan, M. A., van Dokkum, P. G., \& Verheijen, M. A. W. 2001, Science, 293, 1800 